\title{
A NEW MULTI-SPECTRAL THRESHOLD NORMALIZED DIFFERENCE WATER INDEX (MST-NDWI) WATER EXTRACTION METHOD -- A CASE STUDY IN YANHE WATERSHED
}

\author{
Ying Zhou ${ }^{1,2}$, Hongrui Zhao ${ }^{1,2, *}$, Han $\mathrm{Hao}^{3}$, Chaojun Wang ${ }^{1,2}$ \\ ${ }^{1}$ Department of Civil Engineering, Tsinghua University, Beijing 10084, China - (zhouying16@ mails.tsinghua.edu.cn, \\ zhr@tsinghua.edu.cn, wangcj16@mails.tsinghua.edu.cn) \\ ${ }^{2} 3 \mathrm{~S}$ Center, Tsinghua University, Beijing 10084, China \\ ${ }^{3}$ Beijing Institute of Remote Sensing, Beijing 100192, China - (realhao@126.com)
}

Commission III, WG III/1

KEY WORDS: Water Extraction, MST-NDWI, Small Linear River, Remote Sensing, Yanhe Watershed

\begin{abstract}
:
Accurate remote sensing water extraction is one of the primary tasks of watershed ecological environment study. Since the Yanhe water system has typical characteristics of a small water volume and narrow river channel, which leads to the difficulty for conventional water extraction methods such as Normalized Difference Water Index (NDWI). A new Multi-Spectral Threshold segmentation of the NDWI (MST-NDWI) water extraction method is proposed to achieve the accurate water extraction in Yanhe watershed. In the MST-NDWI method, the spectral characteristics of water bodies and typical backgrounds on the Landsat/TM images have been evaluated in Yanhe watershed. The multi-spectral thresholds (TM1, TM4, TM5) based on maximum-likelihood have been utilized before NDWI water extraction to realize segmentation for a division of built-up lands and small linear rivers. With the proposed method, a water map is extracted from the Landsat/TM images in 2010 in China. An accuracy assessment is conducted to compare the proposed method with the conventional water indexes such as NDWI, Modified NDWI (MNDWI), Enhanced Water Index (EWI), and Automated Water Extraction Index (AWEI). The result shows that the MST-NDWI method generates better water extraction accuracy in Yanhe watershed and can effectively diminish the confusing background objects compared to the conventional water indexes. The MST-NDWI method integrates NDWI and Multi-Spectral Threshold segmentation algorithms, with richer valuable information and remarkable results in accurate water extraction in Yanhe watershed.
\end{abstract}

\section{INTRODUCTION}

Water is the key factor to characterize the health condition and stability of the watershed ecosystem. To study the ecological environment of the watershed, the first priority is to accurately analyzing the spatial distribution of water, as well as river length, water area and water amount. Remote sensing technology has the advantages of obtaining a wide range of ground object information simultaneously. With the vigorous development of remote sensing technology, more and more information can be acquired. The water information accurately extracted from remote sensing data can provide decision support for the monitoring and evaluation of the ecological environment, and the investigation and management of water resources. Therefore, the research of water extraction based on remote sensing images is of great significance.

Remote sensing data can be classified in accordance with the spatial resolution, temporal resolution and spectral resolution. Landsat image has been widely recognized as the most widely used medium-resolution, multi-spectral remote sensing data, boasting a spatial resolution that is suitable for ecological environment research at watershed scales, and its spectral resolution can provide efficient support for remote sensing thematic information extraction and classification (Wang et al., 2016). For medium and low-resolution remote sensing images, water extraction methods based on spectral characteristics of ground objects are often used, which can be generally divided into single band method and multi-band method (Xu, H., 2005). Single band method uses the near-infrared band in the remote sensing data for threshold segmentation (Rundquist et al., 1987) to extract water. While the multi-band methods use the difference, ratio or operational relation of reflectivity between two to four bands to enhance water and suppress background for water extraction (Mcfeeters, 1996; Xu, 2005; Pei et al., 2007; Feyisa et al., 2014). For multi band methods, the water index method is relatively convenient and applicable.

In 1996, McFeeters proposed the Normalized Difference Water Index (NDWI), which used the reflection characteristics in the green band and the near-infrared band of the ground objects to distinguish water from vegetation with similar spectral characteristics (Mcfeeters, S. K., 1996); In 2006, Xu proposed the Modified NDWI (MNDWI), in which the near-infrared band of NDWI was replaced by the medium-infrared band, in purpose to reduce the influence of soil/built-up land on water extraction and improve water extraction accuracy in the urban area (Xu, 2005). In 2007, Yan et al. proposed the Enhanced Water Index (EWI) to adopt the green band, near infrared band and medium-infrared band for calculation simultaneously, effectively eliminating the confusion between semi-dry river channels and background objects and improving the extraction of water systems in semi-arid areas (Pei et al., 2007). In 2013, Gudina et al. proposed the Automated Water Extraction Index (AWEI) which utilized five bands including red band, green

* Corresponding author should be addressed to Hongrui Zhao, Email: zhr@tsinghua.edu.cn. 
band, near-infrared band, medium-infrared band and farinfrared band for the construction of the water index, taking full advantage of the information provided by Landsat data to efficiently eliminate the confusion of water extraction due to the deep shadow caused by topography in the mountainous area (Feyisa et al., 2014).

The above methods of water extraction are effective for facial water (such as reservoirs, lakes, etc.) and wide rivers, but cannot separate the background and the water body completely for small linear rivers in Yanhe watershed, and results in low accuracy of water extraction. In this study, a new Multi-Spectral Threshold water extraction method based on Normalized Difference Water Index (MST-NDWI) using Landsat TM image is proposed, which performs better for spatial explicit analysis of small linear rivers over the conventional NDWI method in Yanhe watershed.

\section{STUDY AREA AND DATA SOURCES}

\subsection{Test Sites}

Yanhe River originates from Jingbian County, and it flows from northwest to southeast through Jingbian County, Zhidan County, Ansai District, Baota District and Yanchang County, and finally into the Yellow River near the bank of Liangshui in Yanchang County (Wang, 2014). With a total length of about $286.9 \mathrm{~km}$, the Yanhe watershed is located in the hinterland of the Loess Plateau in northern Shaanxi Province. Its geographical location is $36^{\circ} 23^{\prime}$ to $37^{\circ} 17^{\prime} \mathrm{N}$ and $108^{\circ} 45^{\prime}$ to $110^{\circ} 28^{\prime} \mathrm{E}$ with a total area of approximately $7,725 \mathrm{~km}^{2}$.

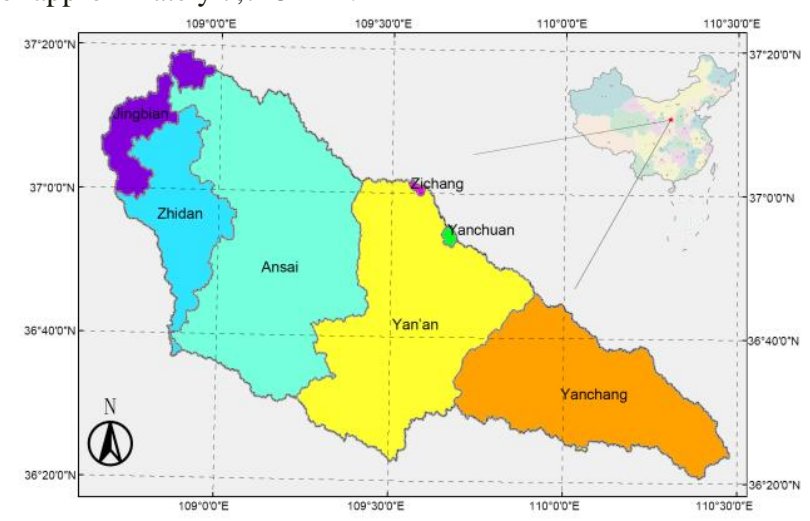

Figure 1. The location and administrative division of the study area.

The topography of the study area is undulating, with the high terrain in northwest and low in southeast. Its water system structure is dendrimer (Chilie et al., 2005), especially in the upper and middle reaches with a river network density of 3.4 $\mathrm{km} / \mathrm{km}^{2}$. The larger tributaries are distributed in this section. The downstream tributaries are short and pinnate.

\subsection{Data Sources}

Remote sensing data used in this paper is the Landsat5 TM image, which was obtained from the Geospatial Data Cloud website (http://www.gscloud.cn/). The product type of all data is L1T, which went through system radiometric correction, ground control point (GCP) geometric correction and topographic correction by DEM. The data projection is UTMWGS84. In view of the relatively limited amount of water in the study area, the TM images of wet periods on the 17th of June, 2010 were selected.

\section{METHODS}

\subsection{Image Pre-processing}

In this study, the fundamental method is to analyze the reflection characteristics of different objects on the TM images. Therefore, obtaining accurate spectral information of ground objects from the images is of vital importance. In order to acquire the spectral information from the image requires atmospheric correction, the Atmospheric correction was applied to the two images using the Fast Line-of-Sight Atmospheric Analysis of Spectral Hypercubes (FLAASH) module in ENVI 5.1. During preprocessing, mosaic and cutting images are also necessary steps to obtain the experimental data.

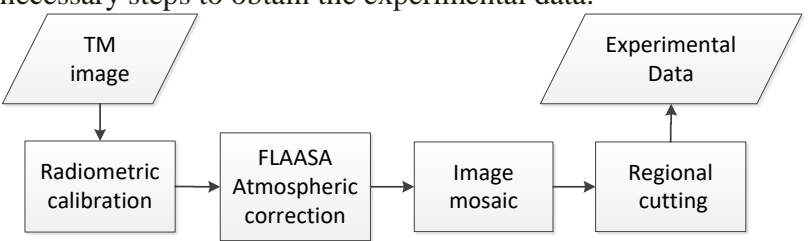

Figure 2. The pre-processing flow chart

\subsection{Water Extraction}

Based on the spectral analysis of remote sensing data, the physical basis of water extraction is the electromagnetic wave reflection characteristics of the ground objects. Considering the inherent nature of the objects, different objects have different reflectivity in different bands. Objects can be classified based on the difference of spectrum characteristic extracted from remote sensing images.

Due to the strong absorbency for incidental energy (solar light), the reflectivity of water is weak, with a tendency to further weaken as the wavelength increases (Xu, 2005). This phenomenon can be clearly seen in Figure 3 that the reflectivity of the spectral curve of water on the TM2 band reaches a maximum, which is significantly higher than that on the TM4 band, that meet the typical spectral characteristics of water (Mcfeeters, 1996). However, for vegetation and built-up land, the reflectivity on the TM4 band is greater than that on the TM2 band, especially for vegetation, and the reflectivity on the nearinfrared band is far greater than that on the green band.

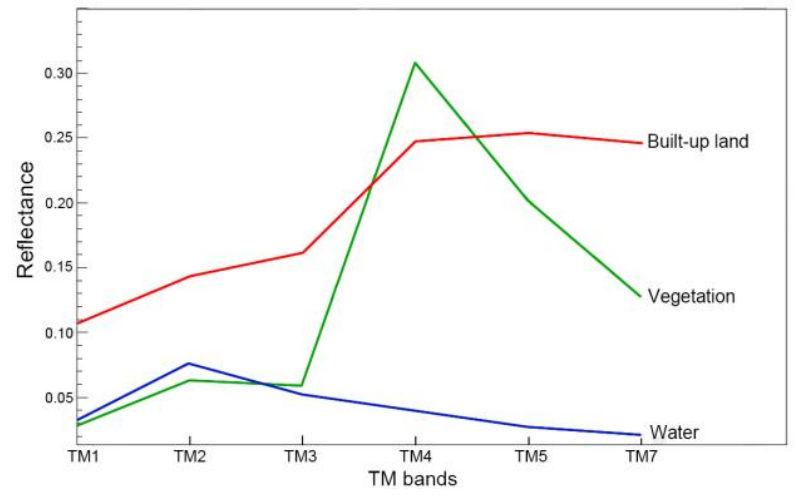

Figure 3. The spectrum extracted from pure pixel samples of built-up land, vegetation and water in the study area, showing the reflection characteristics of different bands on typical objects.

McFeeters (1996) constructed the NDWI water extraction index by using the reflectivity differences of ground objects on the 
green band and near-infrared band. The index is calculated as follows:

$$
N D W I=\frac{G R E E N-N I R}{G R E E N+N I R}
$$

In the formula (1), GREEN refers to the green band, i.e. TM2 band; NIR refers to the near-infrared band, i.e. TM4 band. (Mcfeeters, 1996)

The NDWI index indicates the difference of the variation trend of the spectral curves of different objects, which can be used to distinguish water body and background objects. The conventional method is to judge from the NDWI value, while it's positive, the pixel shall be determined as water, conversely as non-water. In this way, water was extracted.

Applying NDWI to the study area locally to obtain the water extraction results as Figure 4.

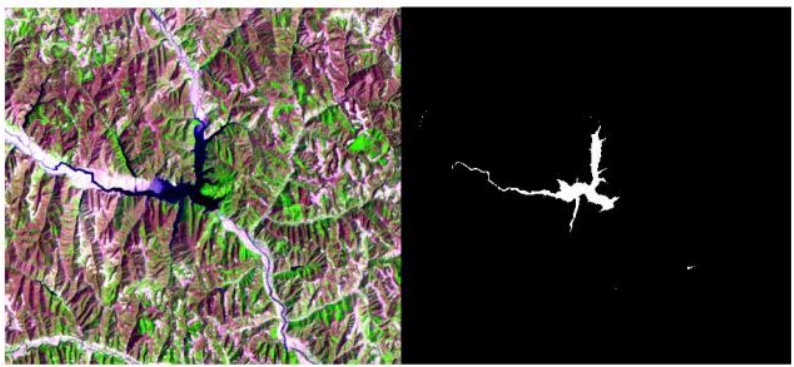

(a) facial water body area, Wangyao reservoir.

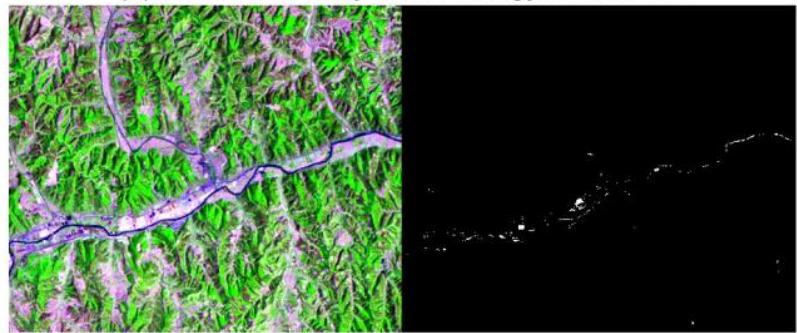

(b) small linear river area, Yan'an subordinate town.

Figure 4. NDWI water extraction results from the study area contrast to the original TM images, displaying the difference in the results of facial water body extraction (a) and small linear river extraction (b).

From the results, the NDWI can enhance water, inhibit vegetation and possess an effective water extraction for facial water bodies such as lakes and reservoirs. While in urban areas, the water extraction is not effective under the condition of small linear rivers. At the same time, built-up background pixels confused in the extraction results.

\subsection{MST-NDWI Water Extraction Method}

In order to understand why NDWI is not effective for extraction of small linear rivers, water with such characteristics has been taken as a class of sample. By analyzing the spectrum of the sampling results, it shows that the linear trend and slop of the spectral curves between built-up land and small linear rivers are similar (Fig.5). The results were negative and the numerical differences were small when calculating the NDWI value. Therefore, a large number of built-up land pixels were determined as water, which would greatly reduce the accuracy of water extraction.
By analyzing the spectral curves of small linear river and builtup land samples, it implies that although the two curves have the same trend of variation, the amplitude difference is obvious. Therefore, the two objects can be segmented by setting the multi-spectral threshold curve appropriately. (Fig.5)

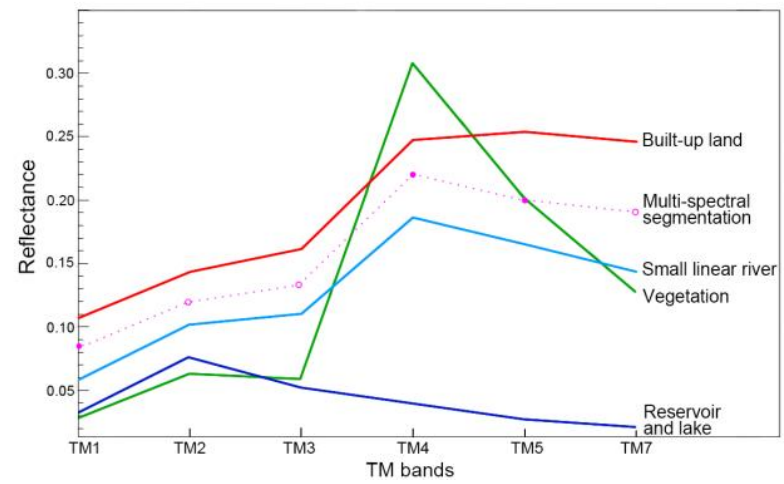

Figure 5. Comparison of the spectral characteristics of typical objects (reservoir and lake, vegetation, small linear river, builtup land) with the proposed multi-spectral segmentation and its potential to better separate built-up land and small linear rivers

Further analysis has been made on the sample data of small linear river with pixel $n=2080$ and sample data of built-up land with pixel $m=2287$ on each band among the six bands. Without losing the generality, a discussion about the X-band has been made. By examining the data, it can be assumed that the two types of ground samples obey the normal distribution, and define the probability densities of small linear river and built-up land are $f\left(x_{r}\right)$ and $f\left(x_{b}\right)$ respectively.

First, use the maximum likelihood estimation to estimate the mean and variance of the two types of samples. Taking the small linear river samples as an example, if the sample set is $\left\{x_{i}^{r}, i=1,2, \ldots, n\right\}$ with a mean value of $\hat{\mu}_{r}$ and a variance of $\hat{\sigma}_{r}^{2}$, the likelihood function is:

$$
L\left(\mu_{r}, \sigma_{r}^{2}\right)=\prod_{i=1}^{n} \frac{1}{\sqrt{2 \pi} \sigma_{r}} \exp \left(-\frac{\left(x_{i}^{r}-\mu_{r}\right)^{2}}{2 \sigma_{r}^{2}}\right)
$$

Take the logarithm of the above equation:

$$
\ln L\left(\mu_{r}, \sigma_{r}^{2}\right)=-\frac{n}{2} \ln (2 \pi)-\frac{n}{2} \ln \left(\sigma_{r}^{2}\right)-\frac{1}{2 \sigma_{r}^{2}} \sum_{i=1}^{n}\left(x_{i}^{r}-\mu_{r}\right)^{2}
$$

Take the partial derivative of the estimated parameter and make it zero:

$$
\left\{\begin{array}{l}
\frac{\partial \ln L\left(\mu_{r}, \sigma_{r}^{2}\right)}{\partial \mu}=\frac{1}{\sigma_{r}^{2}} \sum_{i=1}^{n}\left(x_{i}^{r}-\mu_{r}\right)=0 \\
\frac{\partial \ln L\left(\mu_{r}, \sigma_{r}^{2}\right)}{\partial \sigma_{r}^{2}}=-\frac{n}{2 \sigma_{r}^{2}}+\frac{1}{2 \sigma_{r}^{4}} \sum_{i=1}^{n}\left(x_{i}^{r}-\mu_{r}\right)=0
\end{array}\right.
$$

Finally, obtain the estimated value of distribution parameters. 


$$
\left\{\begin{array}{l}
\hat{\mu}_{r}=\frac{1}{n} \sum_{1}^{n} x_{i}^{r} \\
\hat{\sigma}_{r}^{2}=\frac{1}{n} \sum_{1}^{n}\left(x_{i}^{r}-\hat{\mu}_{r}\right)^{2}
\end{array}\right.
$$

According to the above method, the mean value $\hat{\mu}_{b}$ and the variance $\hat{\sigma}_{b}^{r}$ on the X-band of built-up land samples can be estimated. Then search the segmentation threshold $X_{T}$ within the band interval of $\left[\hat{\mu}_{r}, \hat{\mu}_{b}\right]$,

$$
\underset{X_{T}}{\arg \max }\left(\int_{-\infty}^{X_{T}} f\left(x_{r}\right) d x+\int_{X_{T}}^{+\infty} f\left(x_{b}\right) d x\right)
$$

The segmentation threshold on each band can be obtained by the same method. The following table shows the threshold

\begin{tabular}{|c|c|c|c|}
\hline band & object & Mean & $\begin{array}{l}\text { Segmentation } \\
\text { threshold }\end{array}$ \\
\hline \multirow{2}{*}{ TM1 } & small linear river & 0.0634 & \multirow{2}{*}{0.0867} \\
\hline & built-up land & 0.1093 & \\
\hline \multirow{2}{*}{ TM2 } & small linear river & 0.1016 & \multirow{2}{*}{0.1224} \\
\hline & built-up land & 0.1431 & \\
\hline \multirow{2}{*}{ TM3 } & small linear river & 0.1104 & \multirow{2}{*}{0.1358} \\
\hline & built-up land & 0.1606 & \\
\hline \multirow{2}{*}{ TM4 } & small linear river & 0.1822 & \multirow{2}{*}{0.2169} \\
\hline & built-up land & 0.2443 & \\
\hline \multirow{2}{*}{ TM5 } & small linear river & 0.1642 & \multirow{2}{*}{0.2043} \\
\hline & built-up land & 0.2546 & \\
\hline \multirow{2}{*}{ TM7 } & small linear river & 0.1411 & \multirow{2}{*}{0.1926} \\
\hline & built-up land & 0.2475 & \\
\hline \multicolumn{4}{|c|}{$\begin{array}{l}\text { Table } 1 \text { Segmentation threshold calculated by the two types of } \\
\text { object samples from each band. }\end{array}$} \\
\hline \multicolumn{4}{|c|}{$\begin{array}{l}\text { Use the segmentation thresholds to perform experiments on } \\
\text { each band, the results are displayed in Figure } 6 \text {. As mentioned } \\
\text { above, due to the unsatisfied result of water extraction by } \\
\text { NDWI in the vicinity of Yan'an city, in addition to the analysis } \\
\text { of the entire study area, two key areas (built-up area and airport } \\
\text { area) were selected for threshold segmentation experiment in } 6 \\
\text { bands. The results indicate that the best segmentation effect of } \\
\text { the whole area is obtained from the TM4 band threshold, which } \\
\text { also removes most of the confusion pixels. The thresholds of } \\
\text { TM4 and TM5 have better segmentation effect in airport area, } \\
\text { but poor in built-up area. The thresholds of TM1 and TM7 are } \\
\text { effective for division of built-up area and water bodies. The } \\
\text { thresholds of TM2 and TM3 are relatively weak. Through the } \\
\text { above analysis, the four alternative bands are TM1, TM4, TM5 } \\
\text { and TM7. }\end{array}$} \\
\hline
\end{tabular}
results obtained through search calculations (Table 1)

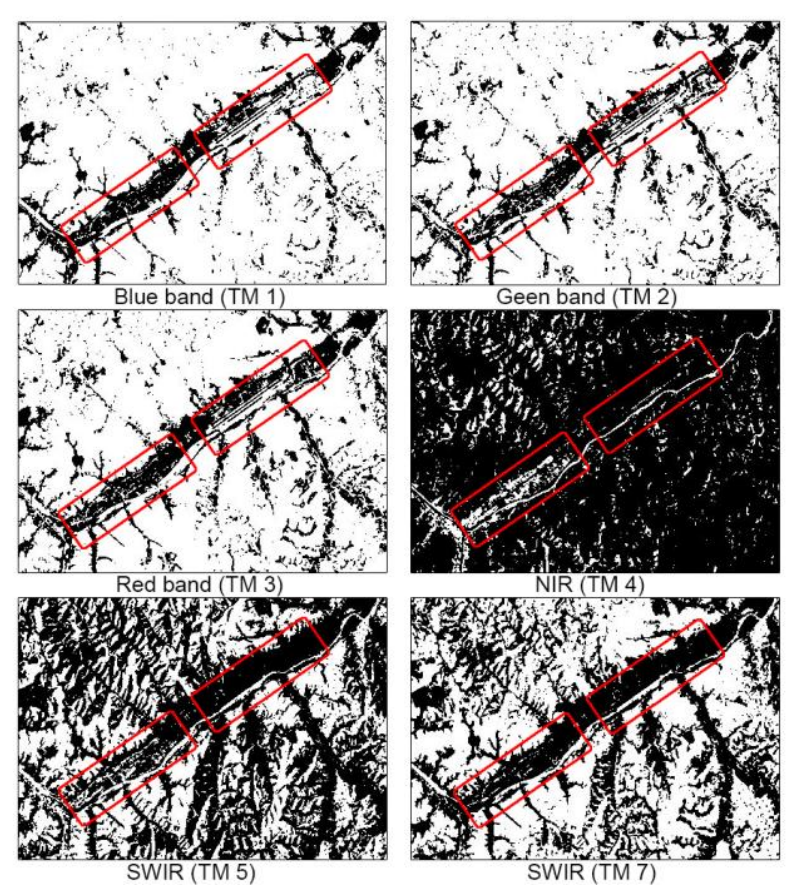

Figure 6. The local results of threshold segmentation in each band, and the segmentation effect of two key regions (built-up area and airport area) in Yan'an city

The physical characteristics on each band of TM and the sensitivity to different ground objects should also be taken into consideration. TM1 refers to the blue band, which can reflect features under shallow water and distinguish soil and vegetation. TM4 refers to near-infrared band, which can distinguish vegetation and water easily because of the strongest reflection area of vegetation and strong absorption area of water. As TM5 is sensitive to the moisture content of vegetation and soil, it is suitable for detecting moisture content of vegetation and soil humidity. TM7 is sensitive to temperature, it is suitable for detecting high-temperature radiation sources, such as monitoring forest fires, volcanic activities.

Considering the segmentation effect of these four thresholds on the entire area, and the theoretical study on the classification of ground objects by the four bands, the TM1 blue band, TM4 near-infrared band and TM5 mid-infrared band were selected to set the threshold. The purpose of threshold segmentation is to retain the pixels of which the value is lower than the three thresholds. In this way, the confusion pixels of built-up land are eliminated. NDWI is then applied to the remaining regions for water extraction, in order to achieve accurate result. The overall flow chart is as follows:

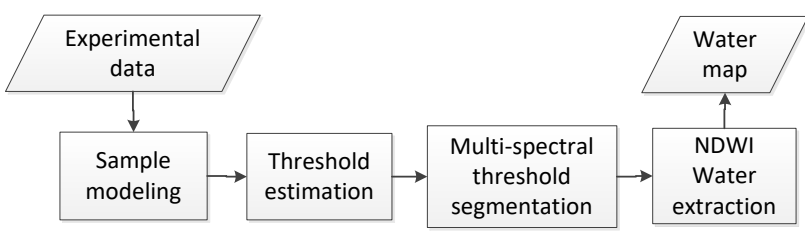

Figure 7. The overall flow chart of the proposed MST-NDWI water extraction method 


\section{RESULTS}

\subsection{Water Extraction Maps}

The MST-NDWI water extraction method was applied to the experimental data of Yanhe watershed in 2010 (Fig.8), and compared with the conventional methods including NDWI, MNDWI, EWI and AWEI water indexes (Fig.9, Fig.10, Fig.11). The water extraction maps were obtained respectively. The analysis of the water extraction effect was based on the different parts (upper, middle and lower reaches) of Yanhe River watershed.

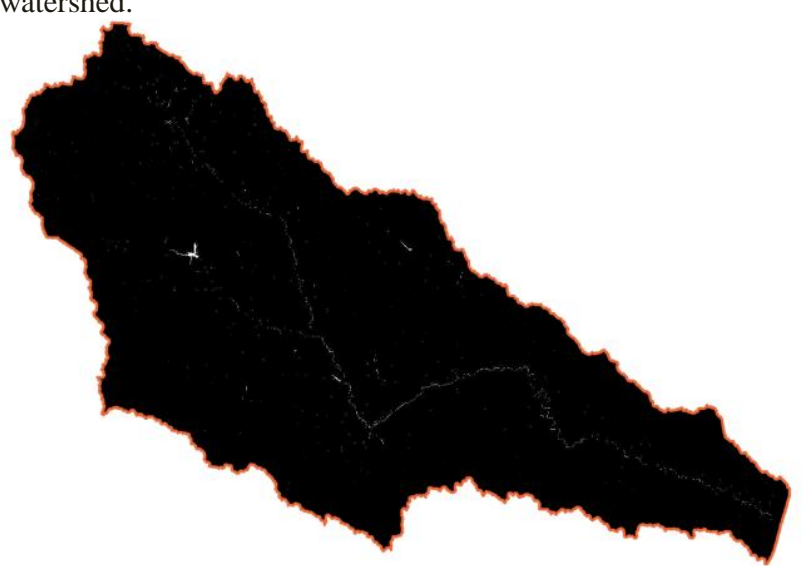

Figure 8. MST-NDWI water extraction map of the entire experimental data of Yanhe watershed

In the upper reaches of Yanhe River (Fig.9), all five methods are effective in medium and large reservoirs. The extraction results of MST-NDWI have clearly showed the Y-shaped trigeminal channel in the upper reaches of Yanhe watershed, which can only be seen in the results of AWEI ${ }_{\text {sh }}$ faintly. However, the Y-shaped river channel can hardly be seen, and the entire water system is vague in the extraction results of NDWI, MNDWI, EWI, and AWEInsh.

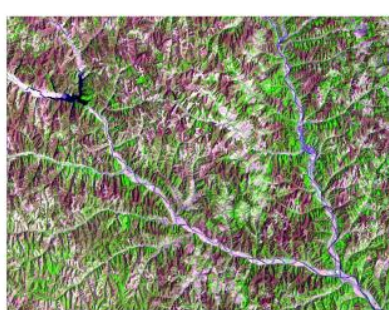

(a) Landsat/TM original image

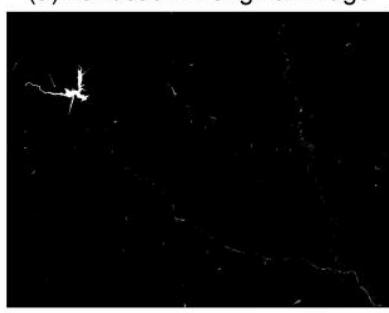

(c) MNDWI method

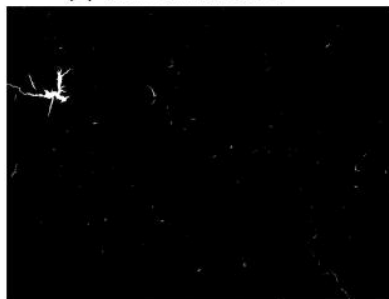

(e) AWEInsh method

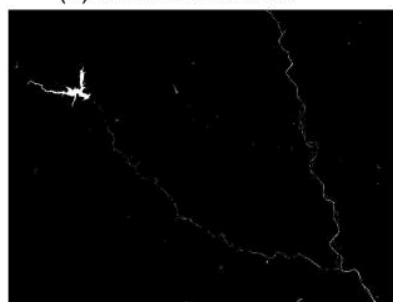

(g) MST-NDWI method

Figure 9. Comparison of Landsat/TM image (a) and conventional Water Index analysis (b)-(f) with the result of

MST-NDWI (g) in the upper reaches of Yanhe River

In the middle reaches of Yanhe River (Fig.10), the results illustrate that the water extracted by AWEInsh method is linear, but it's also intermittent. In addition, on the areas outside the screenshot, it is obvious that many background objects were false-extracted. In the water extraction maps of NDWI, MNDWI, EWI, and AWEI $\mathrm{As}_{\mathrm{sh}}$ methods, the built-up land in Yan'an City, which is a blue-purple region in the original image of Landsat TM, is false-extracted as water, resulting in a chaotic water mapping and vague line feeling. Especially, the results of NDWI, MNDWI and EWI, show that the linear rivers in the east cannot be shown at all. However, the water extracted by MST-NDWI method is a smooth line with uniform thickness and less confusion. 


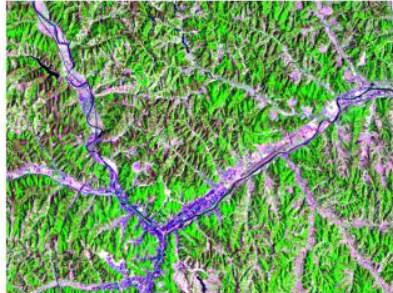

(a) Landsat/TM original image

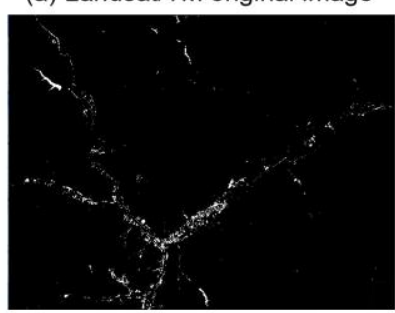

(c) MNDWI method

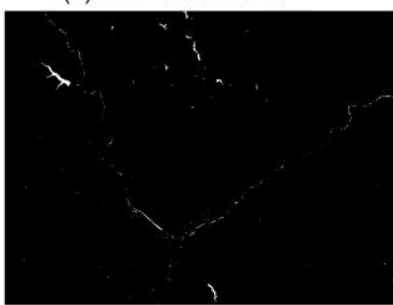

(e) AWEInsh method

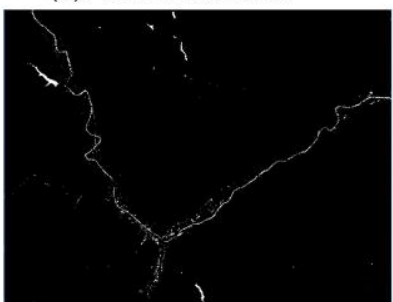

(g) MST-NDWI method

Figure 10. Comparison of Landsat/TM image (a) and conventional Water Index analysis (b)-(f) with the result of MST-NDWI (g) of Yan'an Township in the middle reaches of Yanhe River.

From the water extraction map in the lower reaches of Yanhe River (Fig.11), the results of NDWI, MNDWI and EWI, which is reflected as many weak and inconsistent discrete points, missextracted by these methods. Small linear rivers could reluctantly be seen in the AWEInsh results, but there were large numbers of background objects falsely-extracted outside the river area. Although the clear river lines could be seen in the extraction results of AWEIsh and MST-NDWI, the lines are more consistent and stronger in MST-NDWI.
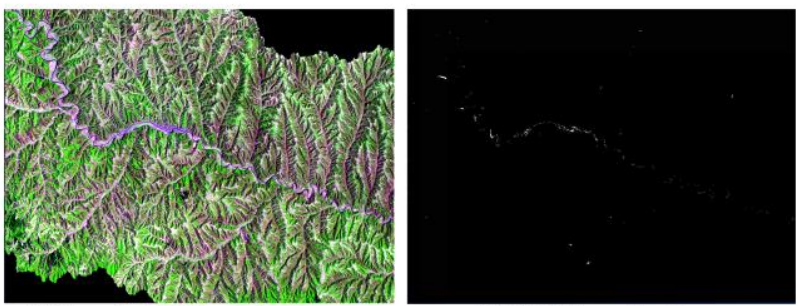

(a) Landsat/TM original image

(b) NDWI method

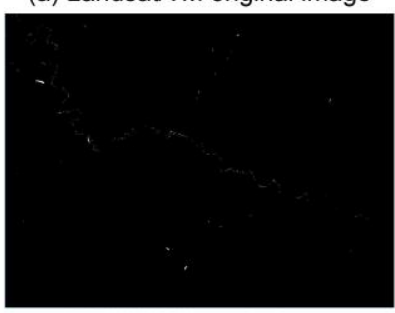

(c) MNDWI method

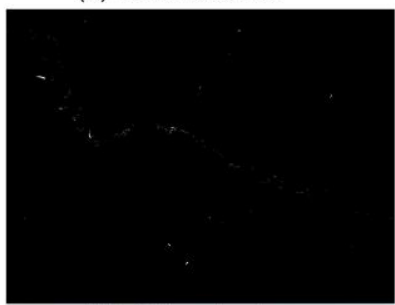

(d) EWI method

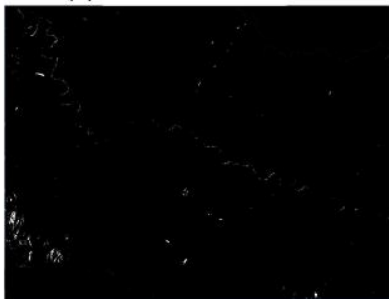

(e) AWEInsh method

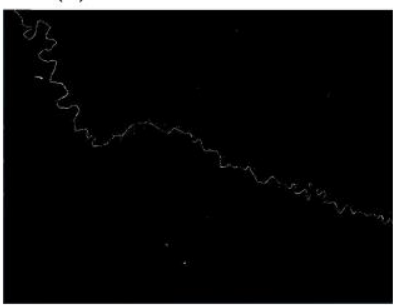

(f) AWEIsh method

(g) MST-NDWI method

Figure 11. Comparison of Landsat/TM image (a) and conventional Water Index analysis (b)-(f) with the result of

MST-NDWI $(\mathrm{g})$ in the lower reaches of Yanhe River.

\subsection{Accuracy}

In the entire experimental area, visual interpretation was used to manual depiction of the contrast TM image, which was used as verification standard. A total of 1500 sample points were selected randomly for accuracy verification, of which 500 samples were in the river area and 200 points in the adjacent area to the river, the remaining 800 points were scattered in the entire areas. In addition to using the sampling results to calculate the overall accuracy of the water extraction methods, the kappa coefficients were calculated using the confusion matrix. The accuracy assessment result is as in Table 2 . 


\begin{tabular}{lllllll}
\hline Methods & $\begin{array}{l}\text { MST- } \\
\text { NDWI }\end{array}$ & NDWI & MNDWI & EWI & AWEI $_{\text {ns }}$ & AWEI $_{\text {sh }}$ \\
\hline $\begin{array}{l}\text { Correct } \\
\text { water points }\end{array}$ & 402 & 270 & 303 & 285 & 317 & 370 \\
$\begin{array}{l}\text { Correct } \\
\text { non-water } \\
\text { points }\end{array}$ & 1012 & 1014 & 1014 & 1015 & 1010 & 1011 \\
$\begin{array}{l}\text { False- } \\
\text { extract } \\
\text { water points }\end{array}$ & 4 & 2 & 2 & 1 & 6 & 5 \\
$\begin{array}{l}\text { Miss-extract } \\
\text { water points }\end{array}$ & 82 & 214 & 181 & 199 & 167 & 114 \\
$\begin{array}{l}\text { Overall } \\
\text { accuracy } \\
\text { (\%) }\end{array}$ & $\mathbf{9 4 . 3}$ & 85.6 & 87.8 & 86.7 & 88.5 & 92.1 \\
\hline \begin{tabular}{l} 
KAPPA \\
\hline
\end{tabular} & $\mathbf{0 . 8 6}$ & 0.63 & 0.69 & 0.66 & 0.71 & 0.81 \\
\hline
\end{tabular}

Table 2. The accuracy assessment results of MST-NDWI and the conventional water indexes such as NDWI, MNDWI, EWI, and AWEI, showing MST-NDWI reached the highest overall accuracy.

The result demonstrated that the MST-NDWI method provides the highest overall accuracy, reaching $94.3 \%$. The advantage lies in reducing the miss-extraction rate by an average of $19.2 \%$ and effectively controlling the false-extraction rate simultaneously.

\section{DISCUSSION}

Conventional water index extraction methods discriminate water and background objects by using the difference of the variation trend among different spectral bands, which implies that the trend of the spectral curves of water and background objects should be different for extraction. However, in Yanhe watershed, the spectral curve trend of small linear river is very similar to that of the built-up lands on TM images. The reason are two folds: On the one hand, due to serious soil erosion in the Yanhe watershed, the total amount of water volume is less, result in semi-arid rivers and high sediment content in rivers, which greatly influence on water information obtained by the remote sensor because of sediment and soil at the bottom of the river. On the other hand, the width of main stream and tributaries are narrow in Yanhe watershed, which result in mixed pixels on remote sensing images, and the pixel spectrum reflected the average of mixed features besides pure water spectrum. In the case of small linear river accounts for a considerable proportion of the Yanhe watershed, simply using the relative indicators defined by conventional water index extraction methods cannot effectively distinguish water from the backgrounds.

The MST-NDWI method starts from the absolute difference between the spectral curves of the two types of objects, uses multi-spectral threshold segmentation to eliminate built-up land pixels similar to those of small linear river. NDWI is then applied to enhance water and weaken the confusion of vegetation and other background objects for accurate water extraction.

\section{CONCLUSION}

This paper analysed the reflection features of water bodies and typical background on the spectral features of Landsat/TM images, and proposed a new MST-NDWI water extraction method. This method established the multi-spectral thresholds using TM1, TM4, TM5 bands to eliminate the confusing builtup land pixels from the image data, makes it more efficient to apply NDWI water extraction method on the remained regions to obtain a more accurate water extraction result under the condition of small water volume and narrow river channel in Yanhe watershed.

Through the evaluation on qualitative results of water extraction experiments, and quantitative analysis on accuracy assessment results, it can be concluded that this new method extends the applicability of NDWI, and can enhance small linear river extraction with better accuracy over other conventional water index extraction methods, which can restore the water system of Yanhe watershed completely and accurately. According to our studies, the proposed method shows great potential and provides a solid foundation for watershed ecosystem studies.

\section{ACKNOWLEDGMENTS}

This study was supported by the National Natural Science Foundation of China "Eco-environment assessment of Yanhe watershed based on temporal-spatial entropy" [Grant number 41571414].

\section{REFERENCES}

Chilie, B., Xing min, M. U., Wang, S. Y., GAO, P., \& Wang, W., 2005. Temporal change analysis of hydrological factors in the yanhe river basin. Bulletin of Soil \& Water Conservation, Vol.25, No.1, pp. 11-14.

Feyisa, G. L., Meilby, H., Fensholt, R., \& Proud, S. R., 2014. Automated water extraction index: a new technique for surface water mapping using Landsat imagery. Remote Sensing of Environment, Vol.140, No.1, pp. 23-35.

Mcfeeters, S. K., 1996. The use of the normalized difference water index (NDWI) in the delineation of open water features. International Journal of Remote Sensing, Vol.17, No.7, pp.1425-1432.

Pei, Y., Zhang, Y. J., \& Yuan, Z., 2007. A study on information extraction of water system in semi-arid regions with the enhanced water index (EWI) and GIS based noise remove techniques. Remote Sensing Information. Vol.6, pp. 62-67.

Rundquist, D. C., Lawson, M. P., Queen, L. P., \& Cerveny, R. S., 1987. The relationship between summer season rainfall events and lake surface area. Journal of the American Water Resources Association, Vol.23, No.3, pp. 493-508.

Wang, C., \& Zhao, H., 2016. The assessment of urban ecological environment in watershed scale. Procedia Environmental Sciences. Vol.36, pp.169-175.

Wang, Z.J., 2014. Characteristics of Vegetation and Erosion Sediment Yield in the Yanhe Watershed. The University of Chinese Academy of Sciences. 
The International Archives of the Photogrammetry, Remote Sensing and Spatial Information Sciences, Volume XLII-3, 2018 ISPRS TC III Mid-term Symposium "Developments, Technologies and Applications in Remote Sensing", 7-10 May, Beijing, China

Xu, H., 2005. A study on information extraction of water body with the modified normalized difference water index (MNDWI).

Journal of Remote Sensing. Vol.9, No.5, pp. 589-595. 